\title{
Transport of Impurity Ions in the Wendelstein 7-AS Stellarator Plasma
}

\author{
Rainer Burhenn, Juergen Baldzuhn, Craig Beidler, Rudolf Brakel, Hartmut Ehmler, \\ Louis Giannone, Peter Grigull, Matthias Hirsch, Jens Knauer, Maciej Krychowiak, \\ Katsumi Ida ${ }^{1}$, Henning Maassberg, Kent McCormick, Ekkehard Pasch, \\ Arthur Weller, W7-AS Team, NBI- and ECRH Group ${ }^{2}$
}

\author{
Max-Planck-Institut für Plasmaphysik, EURATOM-Ass., Wendelsteinstrasse 1, D-17491 Greifswald, Germany \\ ${ }^{1}$ National Institute for Fusion Sciences, Toki, Gifu 509-5292, Japan \\ ${ }^{2}$ Institut für Plasmaforschung, Universität Stuttgart, D-70569 Stuttgart, Germany
}

\begin{abstract}
The impact of global plasma parameters on impurity transport in the stellarator W7-AS was investigated by laser blow-off technique. Both, density and heating power were identified to have a strong influence on impurity confinement $\tau_{\mathrm{I}} \sim \mathrm{n}_{\mathrm{e}}{ }^{1.2} / \mathrm{P}_{\mathrm{ECRH}}{ }^{0.8}$. In spite of stationary conditions at lower densities, an increasing trend for accumulation was observed at plasma densities beyond $5 \cdot 10^{19} \mathrm{~m}^{-3}$ due to reduction of the diffusion coefficients. Up to densities of at least $9 \cdot 10^{19} \mathrm{~m}^{-3}$, launching of electron cyclotron resonance heating (ECRH) power of $1.2 \mathrm{MW}$ is able to counteract the impurity accumulation by deterioration of the impurity confinement with heating power according to the scaling law as given above. In neutral beam injection (NBI) heated plasmas at densities higher than $1 \cdot 10^{20} \mathrm{~m}^{-3}$, long confinement times were observed, often accompanied by loss of density control and degradation of plasma energy due to increasing radiation losses. The installation of island divertor allowed a general extension of the range of accessible densities up to $4 \cdot 10^{20} \mathrm{~m}^{-3}$ : beyond a certain power-dependent threshold density $\left(1.5-2.1 \cdot 10^{20} \mathrm{~m}^{-3}\right)$, the plasma enters the High Density $\mathrm{H}$ mode $(\mathrm{HDH})$ regime and the impurity confinement time drops to values comparable to the energy confinement time. High density plasmas could be sustained quasi-stationary with a low level of impurity radiation. The favourable impurity behavior goes along with a reduction of the inward impurity convection in the core plasma and possible changes in the edge transport. For the characterization of the general impurity behavior in W7-AS plasmas the usual transport models for axisymmetric devices are not sufficient and additional stellarator specific processes have to be considered.
\end{abstract}

Keywords: stellarator, impurity transport, impurity confinement

PACS: 52.55.Hc, 52.25.Vy, 52.25.Fi

\section{INTRODUCTION}

Most of the present day fusion devices still operate in a pulsed plasma operation mode. Only a few machines (e.g. Tore Supra, LHD, Triam-1M, and later Wendelstein 7-X) are equipped with super-conducting coils and therefore capable of significantly extended pulse lengths. This technical property allows the investigation of longterm effects and plasma behavior in complete equilibrium far beyond typical time constants e.g. for current diffusion and thermal equilibration of plasma facing inboard tiles. Going towards steady-state operation, which is basically one of the intrinsic capabilities of stellarators, large efforts have been undertaken in order to solve technical problems concerned with heat load, erosion, deposition and recycling. However, with the achievement of long pulses the remaining question of successful handling of impurity ions and the avoidance of accumulation gain increased importance, in particular for stellarators. The generation of positive electric fields in order to repel impurity ions is investigated in detail at LHD [1,2]. Edge-localized modes (ELM's) as an impurity exhaust mechanism in cases of accumulation are still questionable for future machines due to the intermittently high heat power load introduced to plasma facing inboard tiles. In contradiction to tokamaks, stellarators do not have current driven sawteeth which periodically purifies the plasma core when operating in conventional mode. Introduction of high currents is 
principally possible and useful for many applications (EC current drive) but principally contradictory to the advantageous stellarator specific capability to avoid externally induced currents and, consequently, unfavourable current related phenomena (e.g. MHD aktivity). A cleaning mechanism which is predicted exclusively by neoclassical and Pfirsch-Schlueter (PS) theory itself is the so-called "temperature screening" effect of impurity ions in the ion temperature gradient region. Unlike to tokamaks, this beneficial effect is not expected for nonaxisymmetric devices such as stellarators due to the existence of localized particles which are trapped in numerous local mirrors of the three-dimensional magnetic topology and which introduce additional diffusion mechanisms in the long-mean-free-path ( $1 \mathrm{mfp}$ ) regime [3]. Additionally, the impact of radial electric fields on the diffusion process, is generally expected to be more pronounced in stellarators. Such principal differences to tokamaks require specific attention and should be considered with respect to their consequences for impurity behavior in long-pulse stellarator discharges. For this reason, the detailed study of impurity transport in stellarators and the consideration of stellarator specific transport properties in the existing models is necessary. In order to contribute to this question, confinement times and local transport coefficients were derived at W7-AS under various plasma conditions [4] and the consistency of experimental data with the existing model had been scrutinized.

\section{Experiment}

The advanced modular stellarator Wendelstein 7-AS (major radius $\mathrm{R}=2 \mathrm{~m}$, plasma radius $\mathrm{a}_{\mathrm{p}}<18 \mathrm{~cm}$, magnetic field $\mathrm{B} \leq 2.5 \mathrm{~T}$, nearly shearless vacuum rotational transform selectable between $0.25<1<0.7$ ) is equipped with a set of discrete planar and non-planar coils around the torus in five-fold symmetry according to 5 identical modules. The magnetic field topology in each module is characterized by strongly varying cross sections (triangular and elliptical shape) and is partly drift optimized due to an "effective" elongation of the flux surfaces [5]. The impurity transport was investigated by laser blow-off technique (LBO) mostly with aluminium as tracer material. The temporal behavior of the spectral emission of different ionization states after injection into the flat-top phase of the plasma was observed with vacuum ultraviolet (VUV) spectrometers for the lower ionization states (Al IX, X and XI) and a crystal spectrometer for the highest ionization states (Al XII and XIII), all with central line of sight. The soft-X camera system measured the spatial evolution of the energy integrated tracer radiation behind a $25 \mu \mathrm{m}$ Be-filter with high spatial and temporal resolution. In some discharges, $\mathrm{C}^{6+}$ density profiles at the plasma edge could be provided

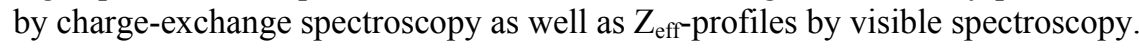

\section{Transport Analysis}

The impurity confinement is characterized by both the confinement time, which is a global quantity representing the total transport across the entire plasma, and local transport coefficients (diffusion coefficient D, convection velocity v). The confinement time is obtained from the temporal decay of spectral lines belonging to the highest ionization states just after the LBO injection. The local transport coefficients were evaluated from the temporal and spatial evolution of the tracer radiation after LBO as measured by the soft-X ray camera system. For this purpose, the radiation evolution has to be converted into an impurity density evolution by an iterative procedure [6] using predictions from the impurity transport and radiation code SITAR [7]. The convective velocity is a strong function of gradients in density and temperature which are often afflicted with certain errors (depending on the profile quality). Hence, for comparison of experimental data with the transport model, we mainly rely on D.

SITAR solves the set of coupled rate equations (continuity equations) for each ionization state $\mathrm{Z}$ of a given tracer impurity species in the presence of a second representative background impurity species in the plasma (e.g., carbon, which is assumed to be the most prominent species due to the numerous power load shields made of this material):

$$
\frac{\partial n_{I}^{z}}{\partial t}=-\frac{1}{r} \frac{\partial}{\partial r}\left(r \Gamma_{I}^{z}\right)+Q_{\text {sources }}+Q_{\sin k s} \quad \text { (1) } \quad \Gamma_{I}^{z}=-D \frac{\partial n_{I}^{z}}{\partial r}+v n_{I}^{z}
$$

The various contributions to the impurity fluxes $\Gamma_{\mathrm{I}}$ have their origin in different driving mechanisms (e.g. gradients in density and temperature, electric fields) and can be sorted and expressed in terms of a diffusive and a convective contribution, The radial profiles of the corresponding local coefficients $\mathrm{D}(\mathrm{r}), \mathrm{v}(\mathrm{r})$ can either be calculated from an implemented neoclassical and PS transport model for axisymmetric devices or freely chosen in order to test simple models. Stellarator specific transport processes are still not implemented in SITAR, except a reduction of the impurity fluxes by a factor of two due to the particle drift optimization of W7-AS compared to a classical stellarator. 


\section{Impact of Stellarator Topology on Impurity Transport}

Under usual plasma conditions at W7-AS, the impurity ions are mostly in the highly collisional PS-regime where the collisional transport dominates the neoclassical one and the impurity transport properties of tokamaks and stellarators, in particular the favourable "temperature screening", are often assumed to be similar. One of the main driving mechanisms for impurity transport is the radial electric field produced exclusively by the background plasma (tracer approximation for impurities). Due to the existence of local mirrors as a consequence of the threedimensional magnetic topology of stellarators, additional collisionality regimes with enhanced transport of the background plasma appear in the $\operatorname{lmfp}$ region $\left(v-, 1 / v^{-}\right.$, and $v^{0.5}$-regime). The enhanced particle losses cause large ambipolar radial electric fields $\mathrm{E}_{\mathrm{r}}$ which in turn reduce the diffusion coefficients of the background plasma, i.e. $\mathrm{D}_{\mathrm{i}}=\mathrm{D}_{\mathrm{i}}\left(\mathrm{E}_{\mathrm{r}}\right)$. This complicated dependence of the fluxes on the electric field has the consequence that $\mathrm{E}_{\mathrm{r}}$ can only be obtained numerically as roots of the non-linear ambipolarity condition $\Gamma_{i}\left(E_{r}, D_{i}\left(E_{r}\right)\right)=\Gamma_{e}\left(E_{r}, D_{e}\left(E_{r}\right)\right)$ with the help of the DKES [8] code in our case. In the case of the so-called ion root $\left(\mathrm{E}_{\mathrm{r}}<0\right)$, which is the most typical one for W7-AS conditions, the simplification $\Gamma_{\mathrm{i}}\left(\mathrm{E}_{\mathrm{r}}\right) \approx 0$ allows for an analytical solution for $\mathrm{E}_{\mathrm{r}}$ as a function of temperature and density gradients of the background ions. $E_{\mathrm{r}}$ can be inserted into the similar expression for the impurity flux. For $\mathrm{T}_{\mathrm{I}}=\mathrm{T}_{\mathrm{i}}, \mathrm{Z}_{\mathrm{i}}=1$ for the background ions and $\mathrm{Z}>>1$ for the impurity ions under consideration follows approximately [3]

$$
\Gamma_{I}^{z}=-n_{I}^{z} D_{I}^{z}\left\{\frac{n_{I}^{z}}{n_{I}^{z}}-Z \frac{E_{r}}{T_{i}}+\delta_{I}^{z} \frac{T_{I}^{\prime}}{T_{I}}\right\} \approx-n_{I}^{z} D_{I}^{z}\left\{\frac{n_{I}^{z}}{n_{I}^{z}}-Z\left(\frac{n_{i}^{\prime}}{n_{i}}+\delta_{i} \frac{T_{i}^{\prime}}{T_{i}}\right)\right\} .
$$

In tokamaks the coefficient $\delta_{\mathrm{i}}$ can be negative (-1/2 for the background ions in the Banana regime) and represents an option to flush out impurities (temperature screening). In stellarators, this coefficient is always positive for all collisionality regimes of the background gas (i.e. inward directed impurity fluxes) [3] except when the background gas itself enters the PS regime, which is the case at the plasma edge of high density plasmas in W7-AS. The electron root $\left(E_{r}>0\right)$, whose field directon is favourable to flush out impurities, could only be realized at low densities in the very central part of the plasma $(\mathrm{r}<5 \mathrm{~cm})$ and resulted in clear impurity density profile effects but was without noticeable effect on the global impurity confinement [9]. The typically larger electric fields in stellarators impose, additionally, strong ExB-forces upon the highly charged impurity ions and might cause (even in the PS regime of the impurity ions) a gradual reduction of their diffusion coefficient down to classical values. It is therefore recommendable to consider stellarator relevant transport properties of ions and impurities self-consistently in future transport codes. The following experimental results were compared with predictions from the existing model (SITAR), but first steps were undertaken in order to account for stellarator specifics as described above.

\section{Impact of Plasma Parameters on Impurity Confinement}

In the following chapter, the sensitivity of impurity transport to variations in the global plasma parameters is studied both in order to explore the actual transport behavior in W7-AS and to investigate, whether the observed trends are compatible with predicted ones.

\section{Scaling of the Impurity Confinement Time}

For ECR heated plasmas $(70 / 140 \mathrm{GHz}$, absorbed at the central resonant magnetic field strength $\mathrm{B}$ of $1.25 / 2.5 \mathrm{~T}$, respectively) a scaling law for the impurity confinement time $\tau_{\mathrm{Al}} \sim \mathrm{a}_{\mathrm{p}}{ }^{2.4} \mathrm{n}_{\mathrm{e}}{ }^{1.2} \mathrm{~B}^{0.3} / \mathrm{P}_{\mathrm{ECRH}}{ }^{0.8}$ (plasma radius $\mathrm{a}_{\mathrm{p}}$, electron density $\mathrm{n}_{\mathrm{e}}$, ECRH heating power $\mathrm{P}_{\mathrm{ECRH}}$ ) was derived for densities below $5 \cdot 10^{19} \mathrm{~m}^{-3}$ [4]. Surprisingly, aside from the pronounced dependence on plasma size, density and the heating power, the magnetic field seems not to play an important role. Additionally, no isotope effect and only a weak dependence on the rotational transform was observed. The existing trends are not in agreement with neoclassical and PS transport and might point to additional presence of turbulent transport - at least locally. For comparison, the energy confinement time in W7-AS scales quite similarly ( $\tau_{\mathrm{E}} \sim \mathrm{a}_{\mathrm{p}}^{2.21} \mathrm{R}^{0.74} \mathrm{n}_{\mathrm{e}}^{0.5} \mathrm{~B}^{0.73} \mathrm{l}^{0.43} / \mathrm{P}_{\text {heating }}{ }^{0.54}$, major radius $\mathrm{R}$ and rotational transform $\mathrm{l}$ ) [10], which might support this assumption. The density and power dependence of the particle diffusion coefficient in the core as well as its density dependence in the scrape-off layer are also found to scale reciprocally with $\tau_{\mathrm{E}}$ and $\tau_{\mathrm{Al}}[11]$. At W7-AS, turbulent transport is known to be reduced with increasing density, which might be compatible with the observed density scaling, but, nevertheless, the power dependence cannot be easily explained by this picture [12]. 


\section{Dependence on Magnetic Field Strength}

The nearly missing dependence of the impurity confinement time on magnetic field strength seems to be striking at first glance, in particular with respect to the improvement in energy confinement which is already abserved when going from 1.25T to full magnetic field 2.5T. Attempts to elucidate the local transport coefficients in two discharges at different field strength [4] (and therefore different ECRH resonance conditions) but otherwise identical plasma parameters turned out to be difficult. In order to be independent of the central resonance conditions in the case of ECR heating, NBI heating was applied. Unfortunately, even due to the sensitivity of the energy confinement on the magnetic field strength but also on differences in the NBI deposition, both discharges (identical line-averaged density and heating power) could not be sufficiently taylored in order to achieve comparable density and temperature profiles. Nevertheless, the diffusion coefficient in the plasma core was indeed found to be much larger at $1.25 \mathrm{~T}$ compared to $2.5 \mathrm{~T}$. At radial positions where the densities in both cases are comparable, differences of a factor 3-4 were derived - almost as expected from theory. However, also the inward convection is enhanced at 1.25T compared to $2.5 \mathrm{~T}$. Consequently, these two terms which determine impurity confinement nearly balance, resulting in a weak B-dependence in the above-mentioned scaling law.

\section{Dependence on Density}

The unfavourable density dependence of the impurity confinement time has severe impact on machine performance at high densities. The increase of confinement time also holds for densities higher than $5 \cdot 10^{19} \mathrm{~m}^{-3}$ into regions beyond the cut-off frequency of the ECRH system $\left(1.2 \cdot 10^{20} \mathrm{~m}^{-3}\right)$ which are accessible only by NBI (Fig.1(a)). The sudden change of confinement at high density when entering the HDH mode is discussed later.

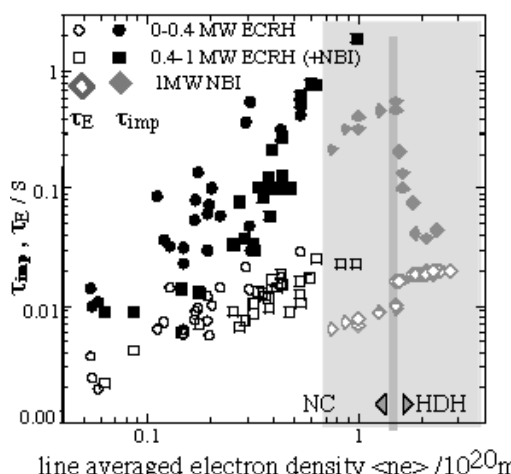

(a)

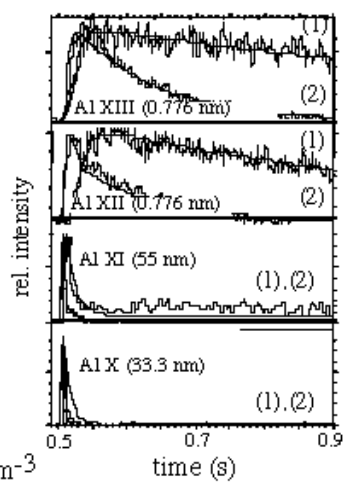

time (s)

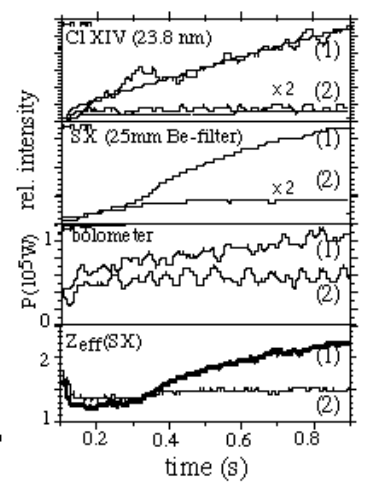

(b)

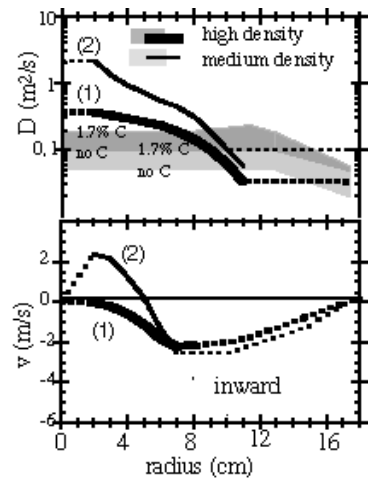

(c)

Figure 1. (a): Energy and impurity confinement times vs. electron density for normal confinement ECRH limiter discharges (NC, white area) and for high density NBI plasmas (shaded area). The generally smaller confinement times for NC NBI plasmas is due to different plasma sizes at different magnetic configurations. The onset of the HDH mode is indicated by the vertical bar. (b): Aluminium-LBO (left) and behaviour of intrinsic radiation (right) in a high-density $\left(7 \cdot 10^{19} \mathrm{~m}^{-3}(1)\right)$ and a medium-density $\left(3.5 \cdot 10^{19} \mathrm{~m}^{-3}(2)\right) 400 \mathrm{~kW}$ ECRH plasma at 2.5T. (c): corresponding transport coefficients (1) and (2) together with model predictions (shaded areas). In regions where the intensity did not allow a reliable derivation of $\mathrm{D}, \mathrm{v}$, dotted lines represent selected values which match the experimental data. The solid lines in (b) represent simulations using the derived D,v in (c). [4].

At low plasma densities, stationary plasmas without accumulation can be established without any problems (Fig.1(b)(2)). However, beyond densities of $5 \cdot 10^{19} \mathrm{~m}^{-3}$ a gradual increase of intrinsic impurity radiation as well as $Z_{\text {eff }}$ was observed (Fig.1(b)(1)) which is consistent with increasing confinement times. This was found [4] to be caused by an overall reduction of the diffusion coefficients at higher density (Fig.1(c)), in particular at the edge, where the low D determines the time constant for reaching stationarity. At low density D is larger and stationarity is achieved on a faster time scale (Fig. 1(c)(2)). Simulations show that also in the case of high density (Fig.1(b)(1)) saturation will be achieved at $1.7 \mathrm{~s}$, i.e. outside the pulse length. This implies, that even in the case of accumulation at high density the plasma can be expected to reach stationary conditions sometime if the radiation losses and the impurity influx, which are directly related, can be kept small in order to avoid a radiation collapse.

In order to rule out the possibility of increasing impurity sources (e.g. due to increasing surface temperature of plasma facing component during the discharge) being responsible for the radiation increase at high density, fluorine 
was puffed into a high density plasma with a constant rate, simulating a constant impurity source [4]. In agreement with the above findings, the fluorine radiation starts to rise throughout the otherwise stationary part of the pulse length, pointing to transport being the responsible mechanism rather than transient sources. Moreover, fluorine density profiles derived from $Z_{\text {eff }}$ profiles at the end of the pulse were peaked with respect to the electron density profile in agreement with code predictions using the corresponding transport coefficients from Fig. 1(c)(1).

\section{Dependence on Heating Power}

The observed deterioration of impurity confinement with heating power was elucidated in two discharges with similar central density $\left(4 \cdot 10^{19} \mathrm{~m}^{-3}\right)$, magnetic field strength $(2.5 \mathrm{~T})$ and edge rotational transform $(\mathrm{l}=0.35)$ but different ECRH heating power $(400 \mathrm{~kW} / 800 \mathrm{~kW})$. It was found that the shorter confinement times and, consequently, the degraded confinement of impurity ions at low density is caused by approximately a factor of 2-3 higher diffusion coefficients throughout the outer $2 / 3$ of the plasma cross section [4]. The change of the diffusion coefficient might be an indication for enhanced turbulent transport at increased heating power, in agreement with the observed power degradation of energy confinement. The beneficial influence of heating power on impurity confinement can be used to counteract the unfavourable dependence of density. In order to explore the possibility to avoid accumulation by application of high ECRH power, discharges ((a) till (d)) at different density $\left(2.5 / 4 / 6 / 7 / 9 \cdot 10^{19} \mathrm{~m}^{-3}\right)$ are heated successively in three steps of heating power (0.4/0.8/1.2 MW) within one pulse. Fig.2 reveals that a plasma with central density of $9 \cdot 10^{19} \mathrm{~m}^{-3}$ (a) can be maintained with a stationary radiation level when using $1.2 \mathrm{MW}$ of ECRH
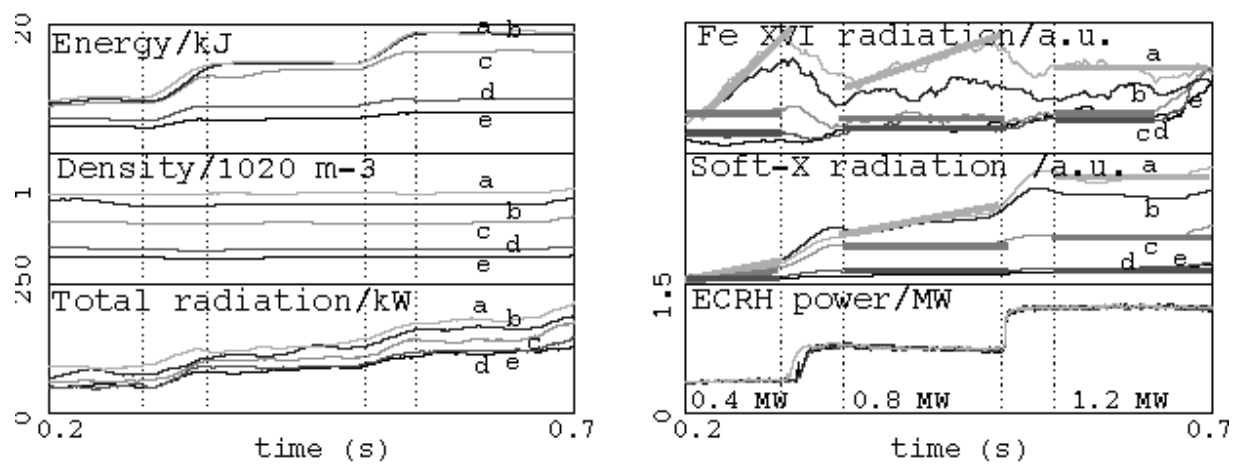

Figure 2: Impurity radiation behavior of plasmas at different density under application of different ECRH heating powers.

heating power. It corresponds to a heating power density of approximately $1 \mathrm{MW} / \mathrm{m}^{3}$. For smaller densities a successively lower power level is sufficient. A principle density limit for this kind of procedure is, of course, given by the cut-off density of $1.2 \cdot 10^{20} \mathrm{~m}^{-3}$ of the ECRH system. For higher densities, non-resonant heating scenarios such as NBI have to be applied with sufficient power. With respect to the previous findings, the principle need of high heating power for achievement of fusion relevant plasma parameters in future machines might simultaneously have a beneficial effect on impurity retention.

\section{Comparision of ECRH and NBI heated Plasmas}

In spite of similar trends concerning the density dependence of impurity confinement (Fig. 1(a)), there are clear differences in the local impurity transport between ECRH and NBI plasmas. In Fig.3 (left, bottom), local transport coefficients of ECRH and NBI plasmas at high density were compared, both with extremely long confinement times and temporally increasing impurity radiation. In ECRH discharges (Fig.3(a)) typically peaked profiles for the diffusion coefficients were found with quite low values at the plasma edge, whereas flat profiles are indicated for NBI plasmas (Fig.3(b)). These properties are in agreement with the evolution of the penetration process of aluminium ions after LBO as measured by the Soft-X camera system (Fig.3, left, top): In ECRH plasmas the time constant of the penetration process seems to be determined by the low diffusion coefficient at the plasma edge. Once the ions passed the edge, they spread out immediately over the entire plasma in agreement with high diffusivity and a certain inward convection. In NBI plasmas the impurity density front moves slowly towards the plasma center while keeping its bump-like shape. This points to a low and flat diffusion coefficient but also to a high inward velocity which drives the front inwards. Of course, it should be mentioned, that these figures show only the radiation - not the impurity density. Nevertheless, they might be useful to point out the principal differences in the penetration 
process, which is almost in agreement with the profiles of the local transport coefficients obtained from the transport analysis. In Fig.3, the experimental data are compared with theoretical predictions (SITAR) assuming both cases, a
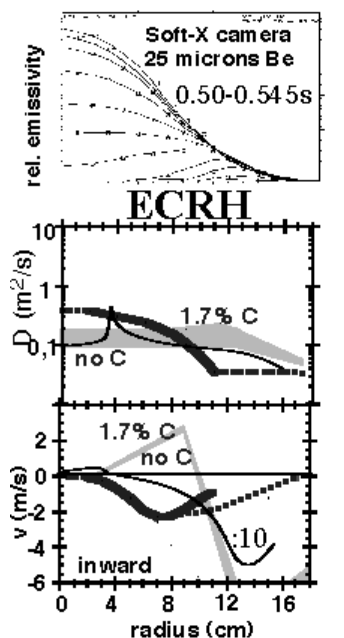

(a)
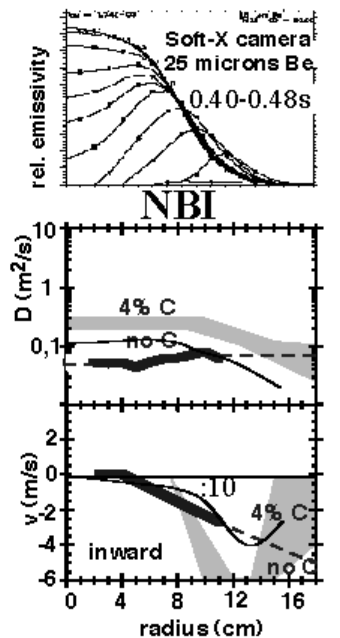

(b)

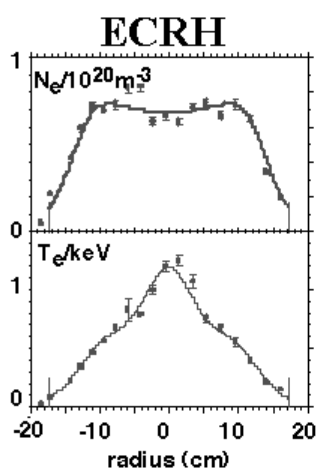

(a)

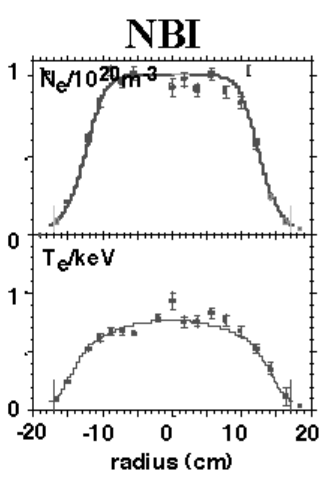

(b)

Figure 3: left,top: LBO penetration evolution into a (a) $500 \mathrm{~kW}$ ECRH plasma (\#38565) and a (b) 500kW NBI plasma, both at high density. Left,bottom: corresponding experimental transport parameters (thick solid line), neoclassical predictions (SITAR, shaded area) with different background impurity concentration and under consideration of stellarator properties (thin line, note: factor of 10 reduced scale only for "stellarator-like" prediction), right: corresponding density and temperature profiles.

pure plasma as well as an additional impurity background concentration of carbon which is compatible with the measured $Z_{\text {eff }}$ values. Obviously, the experimental D's falls below the predicted ones by a factor of 3-8 in the edge region of the high density ECRH plasma, and even more pronounced, over the entire plasma in high density NBI discharges. At present, it is not clear whether this deviation is due to stellarator specific features, which are - as previously stated - not considered in the existing model. As a first step to elucidate the impact of full 3-dimensional magnetic stellarator geometry on transport, the profile of the ambipolar radial electric field produced by transport of the background plasma and its influence on the transport coefficients was calculated numerically by the DKES code. The resulting transport coefficients are presented in Fig.3 and reveal a slight reduction of the diffusion coefficient in direction towards the experimental curve (even more pronounced in the NBI case) - especially at the plasma edge, where the electric fields become large. In this region, also the inward convection is strongly enhanced. Although the predictions still disagree with experiment, the relevance of such effects is visible and subject of ongoing studies [13].

\section{Elmfree H-mode (H*) and High Density H-mode (HDH)}

After W7-AS had been equipped with an island divertor [14], an extended range for high density operation became accessible which resulted in the discovery of the HDH-mode [15,16]. This regime leads to a complete change in impurity transport and allows access to densities up to $4 \cdot 10^{20} \mathrm{~m}^{-3}$ under stationary conditions. Beyond an NBI heating power dependent threshold density of $1.5-2.1 \cdot 10^{20} \mathrm{~m}^{-3}$, the impurity confinement time drops sharply by more than an order of magnitude down to values comparable to the energy confinement time (Fig.1(a)) which, contrarily, slightly rises. Unlike the impurity behaviour in high density normal confinement (NC) plasmas which lead to accumulation and loss of density control, the degraded impurity confinement in HDH now permits access to stationary high density operation at acceptable radiation levels. At the transition from NC to HDH operation, the plasma density profile changes from a peaked one to an extremely flat profile with steep edge gradients whereas the temperature does not change dramatically (Fig.4, left column, middle).

At first glance, the reason for the favourable degradation of impurity confinement in HDH seems to be the reduction of the inward velocity which takes place in the core plasma $(\mathrm{r}<12 \mathrm{~cm})$ at nearly comparable diffusion coefficients (Fig.4, left top). This is in agreement with theory, which predicts the peaked density profiles in NC as a driving mechanism for inward convection. Nevertheless, the price invoked by flat central density profiles in HDH are now steep edge density gradients, which infer a layer of large inward velocity in the gradient region according to theory. Such a "barrier" of high inward convection was indeed experimentally derived [4,17] at W7-AS (Fig.4, left top). The theoretical predictions for D,v (Fig.4, middle column, top) reveal remarkable similarities to the 
experimental data. Unfortunately, the consequences of such a barrier are extremely long confinement times (Fig.4, middle column, bottom (a)(b)), which is in contradiction to the experimental observations. Theory and experiment
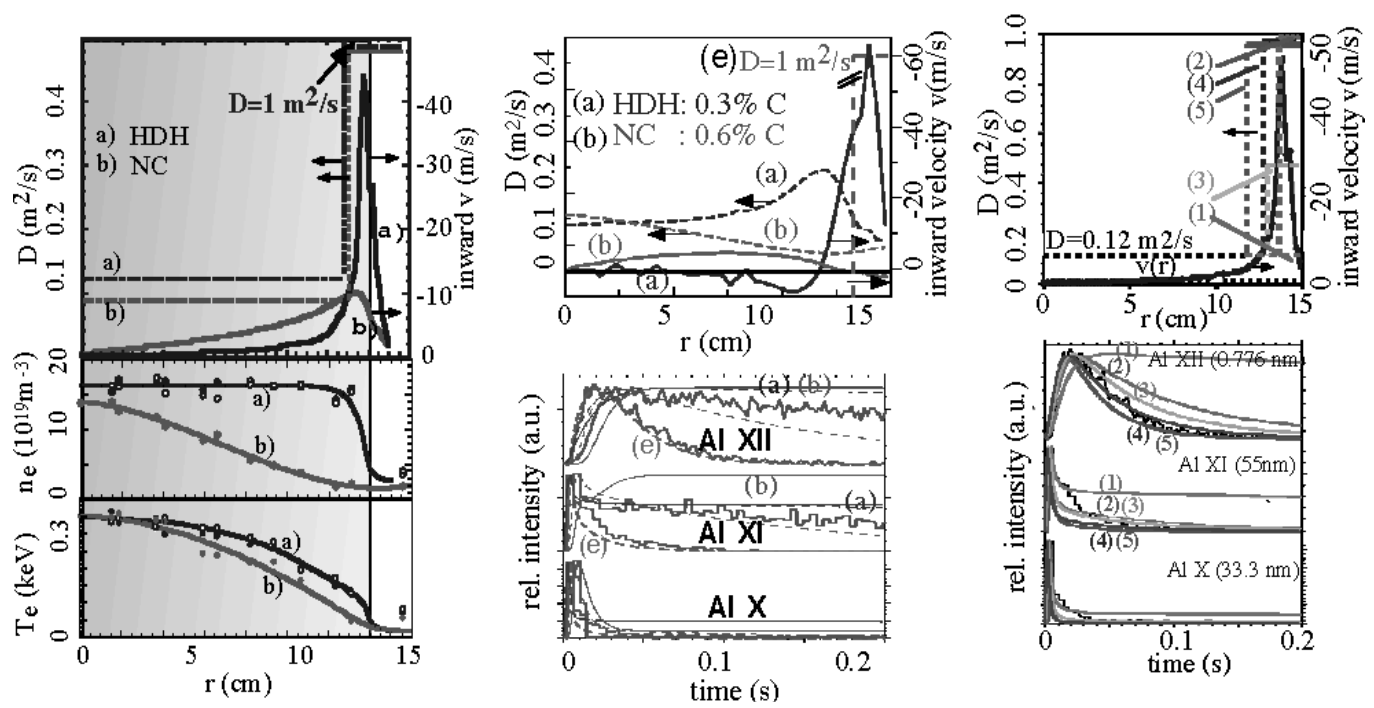

Figure 4: left top: experimental transport parameters for $\mathrm{NC}$ and $\mathrm{HDH}$ plasmas with corresponding density and temperature profiles (left bottom), middle top: model prediction (SITAR) for both cases and (middle,bottom) corresponding LBO simulations (thin lines) compared with experimental traces (thicker lines), right: impact of changes in the edge diffusion on LBO time traces.

could only be brought into agreement (e) by introduction of an artificially high diffusion coefficient in the peripheral region of high inward convection pointing to some kind of turbulent mechanism. The existence of an increased edge diffusion is also supported by measurement of carbon density profiles whose step-like structure at the plasma edge can only be modelled under such assumptions [18]. This indicates, that not only the change of the core density profiles is responsible for the global impurity confinement but also the edge transport. This is additionally confirmed by the totally different confinement properties of $\mathrm{H}^{*}$ [19] mode plasmas, namely long confinement times [4] and the occurrence of impurity accumulation, although density and temperature profile shapes were similar to those in HDH mode. Simulations show, that slight changes of the width, the size and the radial position of the artificially enhanced diffusive layer with respect to the inward convection layer, can reproduce all confinement qualities from $\mathrm{H}^{*}$ to $\mathrm{HDH}$ [4] (Fig.4,right column). Consequently, each modification of transport in this edge localized region can efficiently affect the global impurity confinement. Nevertheless, no clear diagnostic evidence for such enhanced turbulent edge transport (which, moreover, does not effect the energy confinement) was found. At present, the reason for the sudden transition to HDH is not clear and the favourable impurity behaviour not fully understood, but the topic is still subject of on-going investigations [20].

\section{SUMMARY}

In many aspects, the scaling of the global impurity confinement in W7-AS is found to be similar to bulk energy and particle confinement and cannot be explained by the available neoclassical and Pfirsch-Schlueter transport model for axisymmetric devices. This might lead to the assumption, that turbulent transport is additionally involved. Nevertheless, also in plasmas at high density with predominantly neoclassical character concerning the background ions, impurity diffusion coefficients smaller than theoretically predicted were derived. The additional consideration of stellarator specific transport properties, which are introduced by the three-dimensional magnetic topology in stellarators and not considered so far in the existing models for tokamaks, seemed to be important and lead to a somewhat better approximation of the experimental diffusion coefficients. In particular, the "temperature screening" of impurities as present in tokamaks, is not predicted for stellarators and might lead to quite different trends. Although the discrepancy between experiment and predictions could not sufficiently be resolved, the consideration of typical stellarator transport properties appear to be important for a final understanding of the experimental data.

The density and the heating power have the most prominent impact on the impurity confinement at W7-AS, but with different consequences. For densities smaller then $5 \cdot 10^{19} \mathrm{~m}^{-3}$, stationary plasmas with low radiation losses were 
easily maintained. The increasing impurity confinement at higher density causes a growing tendency for impurity accumulation, starting at densities larger then $5 \cdot 10^{19} \mathrm{~m}^{-3}$. For small impurity sources, stationary conditions with acceptable radiation levels are still predicted to be achievable on a time scale which is determined by the low diffusion coefficients and might be somewhat larger than the actual pulse length. In high densitiy NBI plasmas, finally, the plasma energy is noticeably affected by strong radiation losses. However, it could be demonstrated, that the observed deterioration of the impurity confinement due to high heating power (1.2 MW) can prevent accumulation up to densities of $9 \cdot 10^{19} \mathrm{~m}^{-3}$ and might - to a certain degree - also serve as an auxiliary tool to handle the impurity problem in future machines. After the installation of the island divertor in W7-AS, the transition to the $\mathrm{HDH}$ mode occurs beyond a power dependent threshold density and extends the range of accessible densities up to $4 \cdot 10^{20} \mathrm{~m}^{-3}$. In this new regime stationary discharges without impurity accumulation and with low radiation losses were maintained. A part of the favourable confinement degradation is predicted by flattening of the core density profiles in $\mathrm{HDH}$, but the overall confinement seems to be determined mainly by edge transport. This could be shown by comparison of $\mathrm{H}^{*}$ and $\mathrm{HDH}$ mode plasmas which have similar plasma profiles but completely different confinement properties. The formation of the HDH mode as well as the edge processes in the HDH mode are still under investigation.

\section{ACKNOWLEDGMENTS}

The authors would like to thank Prof. Friedrich Wagner and Prof. Thomas Klinger for interesting and helpful discussions, many fruitful suggestions and the support of this work.

\section{REFERENCES}

1. Y. Nakamura et al., "Impurity behaviour in LHD Long Pulse Discharges", Nucl. Fusion, 43, 219 (2003)

2. K. Ida et al., "Control of the radial electric field shear by nodification of the magnetic field configuration in LHD", Nucl. Fusion, 45, 391(2005)

3. H. Maassberg et al., "Density control problems in large stellarators with neoclassical transport", Plasma Phys. Control. Fusion 41, 1135 (1999)

4. R. Burhenn et al., "Impurity transport studies in the Wendelstein 7-AS stellarator", Fusion Science and Technology, 46, 115 (2004)

5. G. Grieger et al., "Physics optimization of stellarators", Phys. Fluids B, 4, 7 (July 1992).

6. R. Burhenn et al., „Derivation of local impurity transport quantities from soft-x radiation evolution during tracer injection at W7-AS“, Rev. Sci. Instrum., 70, 1 (1999)

7. W VII-A Team et al., "Impurity transport in the Wendelstein VII-A stellarator", Nucl. Fusion, 25, 11 (1985)

8. S.P. Hirshman et al., "Plasma transport coefficients for nonsymmetric toroidal confinement systems", Phys. Fluids 29, 2951 (1986)

9. J. Baldzuhn et al., "The role of the radial electric field and plasma rotation for the W7-AS stellarator confinement", ", Proc. $24^{\text {th }}$ Conf. Controlled Fusion and Plasma Physics, Berchtesgaden, Germany, 1997, Europhysics Conference Abstracts, Vol. $21 \mathrm{~A}, 1585-1588$

10. U. Stroth et al., "Energy confinement scaling from the international stellarator database", Nucl. Fusion, 36, 8 (1996)

11. J.P.T. Koponen et al., Pertubative particle transport studies in the W7-AS stellarator", Nucl. Fusion, 40, 365 (2000)

12. U. Stroth et al. "Recent transport experiments in the W7-AS on the stellarator tokamak comparison", Physica Scripta, 51, 655 (1995)

13. C.D. Beidler et al., "Implications of the quasi-neutrality condition for neoclassical transport in stellarators", $15^{\text {th }}$ Int. Stellarator Workshop, Madrid (2005)

14. P. Grigull et al., "Divertor operation in stellarators: results from W7-AS and implications for future devices", Fusion Engeneering and design 66-68 (2003) 49-58

15. K. McCormick et al., "New advanced operational regime on the W7-AS stellarator", Phys. Rev. Lett. 89 (2002) 015001-1

16. R. R. Jaenicke et al., „A new quasi-stationary, very high density plasma regime on the W7-AS stellarator“, Plasma Phys. Control. Fusion, 44, (2002), B193

17. K. Ida et al., "Impurity transport model for the normal confinement (NC) and high density H-mode (HDH) discharges in Wendelstein VII-AS", Plasma Phys. Control. Fusion 45 No.10 (2003) 1931-1938

$18 \mathrm{H}$. Ehmler et al., "Comparative study of intrinsic edge impurities in the W7-AS stellarator during high confinement discharges", Proc. $29^{\text {th }}$ Conf. Controlled Fusion and Plasma Physics, Montreux, Switzerland, 2002, Europhysics Conference Abstracts, Vol. 26B, P4.044

19. K.McCormick et al. "Island divertor experiments on the Wendelstein 7-AS stellarator", J.Nucl. Mater. 313-316 (2002) 1131

20. Y. Igitkhanov et al., "Impurity shielding effect in a H-mode rotation plasma", Proc. $29^{\text {th }}$ Conf. Controlled Fusion and Plasma Physics, Montreux, Switzerland, 2002, ECA Vol. 26B, P-1.106 